\title{
CONTEÚDO E EXPORTAÇÃO DE MICRONUTRIENTES EM ACÁCIA-NEGRA (Acacia mearnsii De Wild.) PROCEDÊNCIA BATEMANS BAY (AUSTRÁLIA) ${ }^{1}$
}

\author{
Marcos Vinicius Winckler Caldeira ${ }^{2}$, Rubens Marques Rondon Neto $^{2}$ e Mauro Valdir Schumacher ${ }^{3}$
}

\begin{abstract}
RESUMO - O objetivo do presente trabalho foi estimar o conteúdo e a exportação de micronutrientes $(\mathrm{Mn}, \mathrm{B}, \mathrm{Cu}$, $\mathrm{Zn}$ e $\mathrm{Fe}$ ) e o $\mathrm{Na}$ nos diferentes componentes de árvores de um povoamento de acácia-negra (Acacia mearnsii De Wild.), procedência australiana Batemans Bay, com 2,4 anos de idade, em Butiá-RS (Brasil). A biomassa total estimada foi de $36.155 \mathrm{~kg} / \mathrm{ha}$, apresentando distribuição de 46,0, 20,0, 19,5, 12,0 e 3,0\%, na madeira do lenho, nas folhas, nos galhos vivos, na casca e nos galhos mortos, respectivamente. A proporção de micronutrientes acumulados na biomassa dos componentes da árvore foram $43,76 \%$ nas folhas, $26,94 \%$ na madeira do tronco, $19,56 \%$ nos galhos vivos, $7,21 \%$ na casca e $2,54 \%$ nos galhos mortos. A quantidade estimada de micronutrientes contidos na biomassa acima do solo foi 10,4 kg/ha, acumulados da seguinte forma: $\mathrm{Na}(58,84 \%), \mathrm{Fe}(21,79 \%), \mathrm{Zn}(9,16 \%), \mathrm{B}$ $(4,09 \%), \mathrm{Mn}(4,59 \%)$ e $\mathrm{Cu}(1,54 \%)$. A casca e a madeira do lenho acumulou $\mathrm{Na}(21,47 \%), \mathrm{Fe}(6,71 \%), \mathrm{Mn}(2,11 \%)$, $\mathrm{Zn}(1,66 \%)$, B $(1,58 \%)$ e $\mathrm{Cu}(0,63 \%)$. A copa (folhas e galhos vivos e mortos) acumularam $\mathrm{Na}(37,36 \%), \mathrm{Fe}$ $(15,07 \%), \mathrm{Zn}(7,49 \%), \mathrm{B}(2,53 \%), \mathrm{Mn}(2,48 \%)$ e $\mathrm{Cu}(0,91 \%)$. A exploração intensiva de áreas com a procedência Batemans Bay gera suspeitas de possíveis ocorrências de deficiências nutricionais de Na nas rotações futuras, tornando necessário o emprego de fertilizantes para manter a produtividade do sítio.
\end{abstract}

Palavras-chave: $\quad$ Micronutrientes, exportação de micronutrientes e Acacia mearnsii.

\section{CONTENT AND EXPORTATION OF MICRONUTRIENTS IN BLACK WATTLE (Acacia mearnsii De Wild.) OF AUSTRALIAN BATEMANS BAY PROVENANCE}

\begin{abstract}
This study aimed to estimate the content and exportation of micronutrients (Mn, B, Cu, Zn e Fe) and $\mathrm{Na}$ in different tree components of black wattle (Acacia mearnsii De Wild.) forest of Batemans Bay Australian provenance, 2.4 years old in Butiá, RS, Brazil. The total biomass found was 36,155 kg/ha, thus distributed: 46.0\%; $20.0 \% ; 19.5 \% ; 12.0 \%$ and $3.0 \%$, spread in the wood of the stem, leaves, live branches, bark and dead branches, respectively. The proportion of the micronutrients accumulated in the biomass of the components were: leaves (43.76\%), wood of the stem (26.94\%), live branches (19.56\%), bark (7.21\%) and dead branches (2.54\%). The estimated micronutrient values in the above-ground biomass were $10.4 \mathrm{~kg} / \mathrm{ha}$, accumulated in $\mathrm{Na}(58.84 \%), \mathrm{Fe}$ (21.79\%), Zn (9.16\%), Mn (4.59\%), B (4.09\%) and $\mathrm{Cu}(1.54 \%)$. The bark and wood accumulated Na values were (21.47\%), Fe (6.71\%), Mn (2.11\%), Zn (1.66\%), B (1.58\%) and $\mathrm{Cu}(0.63 \%)$. The crown (leaves, live and dead branches) accumulated : Na (37.36\%), Fe (15.07\%), Zn (7.49\%), B (2.53\%), Mn (2.48\%) and Cu (0.91\%). The intensive exploration areas of Batemans Bay provenance, signal possible ocurrence of nutritional deficiencies in future rotations, making it necessary to use fertilizer to mantain productivity.
\end{abstract}

Key words: $\quad$ Micronutrients, exportation of micronutrients, Acacia mearnsii.

1 Recebido para publicação em 18.4.2001.

Aceito para publicação em 19.2.2003.

2 Eng. Florestal, Doutorando em Engenharia Florestal na Universidade Federal do Paraná - UFPR, Av. Pref. Lothário Meissner, 3400, Jardim Botânico, 80210-170 Curitiba-PR, <caldeira@floresta.ufpr.br>, <rrondon@floresta.ufpr.br>. ${ }^{3}$ Eng. Florestal, Dr., Prof. Adjunto do Departamento de Ciências Florestais/Centro de Ciências Rurais da Universidade Federal de Santa Maria UFSM, Campus Universitário - Camobi, 97105-900 Santa Maria-RS. 


\section{INTRODUÇÃO}

No Brasil, a acácia-negra (Acacia mearnsii De Wild.) é plantada com fins comerciais principalmente no Estado do Rio Grande do Sul, cobrindo cerca de 100.000 ha. A maioria dessas plantações encontra-se em pequenas propriedades rurais, com participação de mais de 10.000 produtores (Higa et al., 1998). A madeira é utilizada na fabricação de celulose, aglomerado e energia, e da casca é extraído o tanino, que é empregado na indústria farmacêutica, coureira, entre outras.

Grande parte dos povoamentos de acácia-negra é implantada em solos com baixos níveis de fertilidade, e as práticas de uso e manejo do solo normalmente são realizadas de forma incorreta. Sob tais condições os índices de produtividade normalmente são baixos, portanto é indispensável a adoção de práticas de manejo do solo florestal a fim de elevar os níveis de fertilidade e a produtividade dos sítios (Dallago, 2000).

Alguns estudos sobre ciclagem, concentração e exportação de nutrientes da acácia-negra já foram feitos no Brasil, basicamente envolvendo os macronutrientes (Vezzani, 1997; Caldeira, 1998; Caldeira et al., 1999a, 1999b, 1999c, 2000; Pereira et al., 1999, 2000). Entretanto, no que diz respeito às pesquisas realizadas com micronutrientes na acácia-negra até então, têm-se somente os teores de nutrientes nas folhas verificados por Bellote et al. (2000). É clara a necessidade de conhecimentos sobre a distribuição dos micronutrientes nos outros componentes das árvores de acácia-negra, sendo fundamental para estudos de nutrição, ciclagem e exportação dos nutrientes.

O objetivo do presente trabalho foi avaliar as concentrações, os conteúdos e a exportação de micronutrientes em um povoamento de acácia-negra, procedência australiana Batemans Bay, com 2,4 anos de idade, plantado no município de Butiá-RS.

\section{MATERIAL E MÉTODOS}

O presente trabalho foi realizado em fevereiro de 1997, em um povoamento de acácia-negra com 2,4 anos de idade e plantado no espaçamento $1,7 \times 3,0 \mathrm{~m}$, situado na região fisionômica natural do Estado do Rio Grande do Sul, denominada Serra do Sudeste (Escudo Riograndense), município de Butiá-RS. Tal maciço florestal situa-se entre as coordenadas geográficas $30^{\circ} 07^{\prime} 12^{\prime}$ S e $51^{\circ} 57^{\prime} 45^{\prime}$ 'W e a uma altitude média de $35 \mathrm{~m}$.
O clima da região é o Cfa, segundo a classificação de Köppen, ou seja, clima subtropical (Moreno, 1961). A temperatura média anual desta região é de 18 a $19^{\circ} \mathrm{C}$, com temperaturas médias máximas e mínimas no ano de 24 e $14{ }^{\circ} \mathrm{C}$, respectivamente. A precipitação média anual é de $1.400 \mathrm{~mm}$ (Ipagro, 1989).

O solo da região pertence à Unidade de Mapeamento São Jerônimo, classificado como Argissolo VermelhoEscuro (Embrapa, 1999), com textura argilosa e relevo ondulado. Os solos dessa Unidade são, em sua maioria, profundos, bem drenados, avermelhados, franco-argilosos ou argilosos com cascalhos, porosos e desenvolvidos a partir de granito. São também fortemente ácidos, com baixa saturação de alumínio e soma de bases e teores baixos de matéria orgânica (Brasil, 1973).

Para estimar a biomassa e o conteúdo de micronutrientes da parte acima do solo da acácia-negra, selecionaram-se nove árvores, procedentes de Batemans Bay,

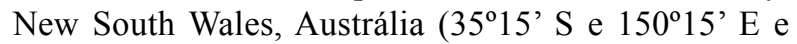
altitude de 20 m s.n.m.). A seleção das árvores para este estudo foi a partir dos dados do inventário florestal, que permitiu agrupá-las em nove classes diamétricas, retirando uma árvore por cada classe.

$\mathrm{Na}$ parte intermediária da copa das árvores, nos quatro pontos cardeais, foram coletados cerca de $300 \mathrm{~g}$ de folhas para realização de análise nutricional. Os galhos foram classificados como vivos e mortos, devendo ser ressaltado que todas as folhas dos galhos vivos foram colhidas.

A massa fresca total das folhas, dos galhos vivos e mortos, da casca e da madeira do tronco das nove árvores foi determinada no campo. De cada um desses componentes foram retiradas subamostra para aferição da massa fresca no campo. Posteriormente, cada subamostra foi acondicionada em sacos plásticos identificados e levada ao Laboratório de Ecologia Florestal do Departamento de Ciências Florestais da Universidade Federal de Santa Maria, onde cada componente foi embalado em sacos de papel-pardo e colocados em estufa com circulação forçada $\left(75^{\circ} \mathrm{C}\right)$, até atingir peso constante.

A madeira do tronco foi amostrada, retirando-se um disco de 5,0 cm de espessura na metade da altura total da árvore (Yong \& Carpenter, 1976). Deste disco, separaram-se a casca e a madeira e registrou-se a massa fresca de cada componente. Após a secagem em estufa as amostras desses dois componentes foram picadas, para facilitar a moagem. As amostras foram moídas e passadas 
em peneira com malha de $1,0 \mathrm{~mm}$. Depois que todos os componentes da árvore foram moídos, retirou-se uma amostra, para realização das análises químicas.

Foram feitas as determinações de Na, Fe, Mn, Zn, $\mathrm{B}$ e $\mathrm{Cu}$, em cada componente da árvore, seguindo as metodologias propostas por Tedesco et al. (1995). As análises foram realizadas no Laboratório de Análise de Solos do Departamento de Solos da Faculdade de Agronomia da Universidade Federal do Rio Grande do Sul.

$\mathrm{O}$ estoque de micronutrientes contidos em todos os componentes da biomassa acima do solo, em $\mathrm{kg} / \mathrm{ha}$, foi obtido a partir da biomassa estimada por Caldeira (1998) e da concentração dos micronutrientes em cada componente. A soma dos valores dos micronutrientes para cada componente da biomassa acima do solo forneceu o seu conteúdo total em kg/ha.

\section{RESULTADOS E DISCUSSÃO}

A Figura 1 mostra a contribuição porcentual de cada componente na biomassa total acima do solo da acácianegra, que foi estimada em $36.155 \mathrm{~kg} / \mathrm{ha}$. A distribuição da biomassa em ordem decrescente foi: lenho $>$ folhas $>$ galhos vivos $>$ casca $>$ galhos mortos. No mesmo local do presente estudo, Caldeira et al. (1999a, 2000) encontraram $41.107 \mathrm{~kg} / \mathrm{ha}$ e $19.475 \mathrm{~kg} / \mathrm{ha}$ de biomassa acima do solo para as procedências australianas Lake Geoge Bunge Dore e Bodalla, respectivamente, aos 2,4 anos de idade.

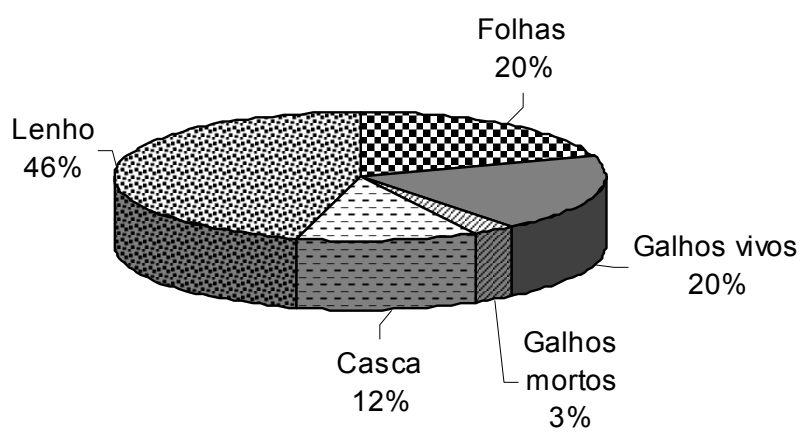

Figura 1 - Distribuição da biomassa acima do solo da acácianegra (Acacia mearnsii De Wild.) procedência australiana Batemans Bay, com 2,4 anos de idade, em Butiá-RS.

Figure 1 - Distribution of above-ground biomass of black wattle (Acacia mearnsii De Wild.) of Batemans Bay Australian provenance, 2.4 years old, in Butiá, RS, Brazil.
O Quadro 1 mostra os resultados do conteúdo, da quantidade média e da porcentagem dos micronutrientes acumulados em cada componente da biomassa acima do solo da acácia-negra. Verificou-se que cerca de $43,76 \%$ dos micronutrientes acumulam-se nas folhas, $26,94 \%$ na madeira, $19,56 \%$ nos galhos vivos, $7,21 \%$ na casca e $2,54 \%$ nos galhos mortos. Portanto, na copa (folhas e galhos vivos e mortos) concentra-se $65,86 \%$ do total de micronutrientes que se acumularam na biomassa acima do solo. Nas folhas encontra-se a maioria das células vivas da árvore, que tendem a acumular maiores quantidades de nutrientes, em função dos processos de transpiração e fotossíntese (Kramer \& Kozlowski, 1979). Comparando os resultados dos conteúdos de $\mathrm{Cu}, \mathrm{Fe}, \mathrm{Mn}$ e $\mathrm{Zn}$ acumulados nas folhas da acácia-negra obtidos com os resultados encontrados por Bellote et al. (2000), em um povoamento de acácia-negra com 3 anos de idade, observam-se semelhanças nos resultados. Acredita-se que o motivo para tal constatação seja o fato de se tratar da mesma espécie, de idades próximas e da mesma metodologia de coleta das folhas para análises químicas. Segundo van den Driessche (1984), Koehler (1989) e Bellote (1990), o acúmulo de nutrientes em várias partes da planta varia consideravelmente com a espécie, a procedência, a idade, a posição das folhas, a época do ano, o sítio e a concentração do nutriente no solo.

A estimativa do estoque de micronutrientes acumulados em todos os componentes da biomassa da acácianegra acima do solo foi de $10,4 \mathrm{~kg} / \mathrm{ha}$. Deste total, a contribuição de cada nutriente analisado foi: $\mathrm{Na}$ (58,84\%), Fe (21,79\%), Zn (9,16\%), Mn (4,59\%), Cu $(1,54 \%)$ e B (4,09\%). Em valores absolutos, o $\mathrm{Na}$ e o $\mathrm{Fe}$ representam cerca de $8,4 \mathrm{~kg} / \mathrm{ha}$, ou seja, $80,63 \%$ do total de nutrientes acumulados na biomassa das árvores acima do solo. Em florestas naturais de bracatinga (Mimosa scabrella Benth.), Baggio (1994) encontrou média de $10,06 \mathrm{~kg} / \mathrm{ha}$ de micronutrientes acumulados na biomassa acima do solo, tendo sido constatadas semelhanças nas proporções de acúmulo dos nutrientes entre as duas leguminosas.

A elevada contribuição do Na em relação aos demais nutrientes pode ter sido causada pela diferença de capacidade de absorção deste elemento das raízes e pela translocação para as partes novas da planta, devendo ser destacado que, substancialmente, tal fato difere entre genótipos de uma mesma espécie (Marschner, 1997). A alta absorção de Na por acácia-negra pode ter ocorrido em função de este elemento estar sendo absorvido no 
Quadro 1 - Resultados do conteúdo médio, da quantidade média e da proporção de micronutrientes nos diferentes componentes da biomassa acima do solo de acácia-negra (Acacia mearnsii De Wild.) procedência australiana Batmans Bay, com 2,4 anos de idade no município de Butiá-RS

Table 1 - Results of average content, average amount and ratio of micronutirents in the different components of above-ground biomass of black wattle (Acacia mearnsii De Wild.) of Batemans Bay Australian provenance, 2.4 years old, in Butiá, RS, Brazil

\begin{tabular}{|c|c|c|c|c|c|c|c|c|c|c|c|c|c|c|c|c|c|c|}
\hline \multirow{3}{*}{ Componente } & \multicolumn{18}{|c|}{ Nutriente } \\
\hline & \multicolumn{3}{|c|}{$\mathrm{Mn}$} & \multicolumn{3}{|c|}{$\mathrm{Na}$} & \multicolumn{3}{|c|}{ B } & \multicolumn{3}{|c|}{$\mathrm{Cu}$} & \multicolumn{3}{|c|}{$\mathrm{Zn}$} & \multicolumn{3}{|c|}{$\mathrm{Fe}$} \\
\hline & $\mathrm{Mg} / \mathrm{kg}$ & $\mathrm{kg} / \mathrm{ha}$ & $\%$ & $\mathrm{Mg} / \mathrm{kg}$ & $\mathrm{kg} / \mathrm{ha}$ & $\%$ & $\mathrm{Mg} / \mathrm{kg}$ & $\mathrm{kg} / \mathrm{ha}$ & $\%$ & $\mathrm{Mg} / \mathrm{kg}$ & $\mathrm{kg} / \mathrm{ha}$ & $\%$ & $\mathrm{Mg} / \mathrm{kg}$ & $\mathrm{kg} / \mathrm{ha}$ & $\%$ & $\mathrm{Mg} / \mathrm{kg}$ & $\mathrm{kg} / \mathrm{ha}$ & $\%$ \\
\hline Folhas & 29,67 & 0,213 & 2,05 & 345,44 & 2,486 & 23,92 & 18,33 & 0,132 & 1,27 & 8,28 & 0,059 & 0,57 & 90,78 & 0,653 & 6,28 & 139,78 & 1,006 & 9,68 \\
\hline Galhos vivos & 5,26 & 0,037 & 0,36 & 178,44 & 1,258 & 12,10 & 16,89 & 0,119 & 1,14 & 4,24 & 0,030 & 0,29 & 15,67 & 0,110 & 1,06 & 67,89 & 0,479 & 4,61 \\
\hline Galhos mortos & 7,80 & 0,008 & 0,08 & 135,44 & 0,140 & 1,35 & 11,67 & 0,012 & 0,12 & 5,43 & 0,006 & 0,06 & 15,94 & 0,016 & 0,15 & 79,44 & 0,082 & 0,79 \\
\hline Casca & 14,33 & 0,061 & 0,59 & 60,67 & 0,259 & 2,49 & 15,44 & 0,066 & 0,63 & 2,58 & 0,011 & 0,11 & 12,69 & 0,054 & 0,52 & 69,89 & 0,298 & 2,87 \\
\hline Madeira & 9,51 & 0,158 & 1,52 & 118,78 & 1,973 & 18,98 & 5,78 & 0,096 & 0,92 & 3,28 & 0,054 & 0,52 & 7,16 & 0,119 & 1,14 & 24,11 & 0,040 & 3,85 \\
\hline Total & 66,57 & \begin{tabular}{|l|l|}
0,477 \\
\end{tabular} & 4,59 & 838,17 & 6,116 & 58,84 & 68,11 & 0,425 & 4,09 & 23,81 & 0,160 & 1,54 & 142,24 & 0,952 & 9,16 & 381,11 & 2,265 & 21,79 \\
\hline
\end{tabular}

lugar do K, mas tal fato precisa ser mais estudado. Neste sentido, foi observado que as procedências de acácianegra Batemans Bay e Bodalla, depois do N, possuem as maiores quantidades de $\mathrm{K}$ na biomassa acima do solo (Caldeira et al., 2000, 2001).

Segundo Marschner (1997), o papel do Na na nutrição mineral de plantas é substituir o K em determinadas funções fisiológicas, como: funções específicas no meristema, na expansão de tecidos e células, no balanço de água das plantas e no aumento do número de estômatos por unidade de área. Em determinadas espécies, 95\% do $\mathrm{K}$ presente no substrato pode ser substituído por $\mathrm{Na}$.

A casca e a madeira do lenho da acácia-negra acumularam 34,14\% do total de micronutrientes, cujos componentes das árvores são extraídos das florestas. Caso o povoamento fosse explorado com 2,4 anos de idade, cerca de $3,549 \mathrm{~kg} / \mathrm{ha}$ de micronutrientes seriam exportados do sítio. Porém, deve-se lembrar que a rotação da acácianegra ocorre entre 7 e 9 anos. Segundo Lima (1996), o corte das árvores mais jovens pode, genericamente, remover mais nutrientes do que o corte em idades mais avançadas.

Em termos porcentuais, a participação dos micronutrientes da casca e da madeira da acácia-negra sobre a concentração total $(10,4 \mathrm{~kg} / \mathrm{ha})$ de nutrientes acumulados foi: $\mathrm{Na}(21,47 \%), \mathrm{Fe}(6,71 \%), \mathrm{Mn}(2,11 \%), \mathrm{Zn}(1,66 \%)$, $\mathrm{B}(1,58 \%)$ e $\mathrm{Cu}(0,63 \%)$. Em valores absolutos, o Na e o Fe também foram os elementos que mais se acumularam na casca e no lenho da madeira, 2,232 e 0,698 kg/ha, respectivamente. $\mathrm{Na}$ seqüência, têm-se os demais micronutrientes: Mn (0,219 kg/ha), Zn (0,173 kg/ha),
$\mathrm{B}(0,162 \mathrm{~kg} / \mathrm{ha})$ e $\mathrm{Cu}(0,065 \mathrm{~kg} / \mathrm{ha})$. Poggiani et al. (1983) constataram que a madeira do lenho e a casca de Eucalyptus saligna, com 8 anos de idade, acumularam $14,61 \mathrm{~kg} / \mathrm{ha}$ de micronutrientes, sendo o $\mathrm{Mn}$ e Fe os elementos mais exportados pela colheita.

A copa (folhas e galhos vivos e mortos) da acácianegra acumulou cerca de $65,86 \%$ do total de micronutrientes. Supondo um sistema de exploração que deixasse no sítio esses componentes da árvore, cerca de $6,846 \mathrm{~kg} / \mathrm{ha}$ de micronutrientes permaneceriam no sítio. Caldeira et al. (2000), investigando o conteúdo de macronutrientes na biomassa aérea da acácia-negra de procedência Bodalla, também com 2,4 anos de idade, constataram que as copas acumulam $70,20 \%$ do total de nutrientes.

Do total de nutrientes acumulados nas árvores de acácia-negra, a participação porcentual dos nutrientes contidos na copa foi: $37,36 \%$ de $\mathrm{Na}, 15,07 \%$ de Fe, 7,49\% de $\mathrm{Zn}, 2,53 \%$ de B, 2,48\% de $\mathrm{Mn}$ e $0,91 \%$ de $\mathrm{Cu}$. Novamente, o Na e o Fe foram os nutrientes que mais se acumularam na copa, ou seja, 3,884 e 1,567 kg/ha, respectivamente, seguidos do $\mathrm{Zn}(0,779 \mathrm{~kg} / \mathrm{ha}), \mathrm{B}(0,263 \mathrm{~kg} / \mathrm{ha})$, $\mathrm{Mn}(0,258 \mathrm{~kg} / \mathrm{ha})$ e $\mathrm{Cu}(0,095 \mathrm{~kg} / \mathrm{ha})$. Nas copas (acículas e galhos) de Pinus taeda L. com 7 anos idade, Valeri et al. (1989) encontraram $6,01 \mathrm{~kg} / \mathrm{ha}$ de micronutrientes, sendo o Mn e o Fe os que mais se acumularam. Os elevados conteúdos de micronutrientes nas copas da acácianegra mostram que esse componente vegetal é muito importante na ciclagem de nutrientes. Portanto, a adoção de sistemas de exploração que deixem no sítio a copa das árvores pode contribuir em parte com o fornecimento de macro e micronutrientes para as rotações subseqüentes. 


\section{CONCLUSÕES}

Com os resultados do presente estudo pode-se concluir que $65,86 \%$ do total de micronutrientes acumulados na biomassa acima do acácia-negra está na copa (folhas e galhos verdes e mortos), apesar de representar $42,40 \%$ da biomassa total das árvores. $\mathrm{O} \mathrm{Na}$ foi o elemento que mais se acumulou na casca e no lenho da madeira, cerca de $21,47 \%$ do conteúdo total de nutrientes contidos na biomassa total. Portanto, a exploração intensiva de áreas com a procedência Batemans Bay gera suspeitas de possíveis ocorrências de deficiências nutricionais de $\mathrm{Na}$ nas rotações futuras, o que torna necessário o emprego de fertilizantes para manter a produtividade do sítio.

\section{REFERÊNCIAS BIBLIOGRÁFICAS}

BAGGIO, A. J. Estudio sobre el sistema agroforestal tradicional de la bracatinga (Mimosa scabrella Benth.) en Brasil productividad, manejo de residuos y elaboracion de compost. 1994. 242 f. Tese (Doutorado em Ingeniero de Montes) - Escuela Técnica Superior de Inginieros de Montes, Madrid, 1994.

BELLOTE, A. F. J. Suprimento de nutrientes minerais e crescimento de plantações adubadas de Eucalyptus grandis nos cerrados do Estado de São Paulo. 1990. 166 f. Tese (Doutorado em Doutorado em Ciências Florestais) Universidade de Fraiburg, Fraiburg, 1990.

BELlote, A. F. J.; SILVA, H. D.; DEDECEK, R. A. Teores de macro e micronutrientes em acácia-negra, com três anos de idade, plantada no Estado do Rio Grande do Sul. In: FERTBIO 2000 - BIODINÂMICA DO SOLO; REUNIÃO BRASILEIRA DE FERTILIDADE DO SOLO E NUTRIÇÃO DE PLANTAS, 24., 2000; REUNIÃO BRASILEIRA SOBRE MICORRIZAS, 8., 2000; SIMPÓSIO BRASILEIRO DE MICROBIOLOGIA DO SOLO, 6., 2000; REUNIÃO BRASILEIRA DE BIOLOGIA DO SOLO, 3., 2000. Resumos Expandidos... Santa Maria: SBCS/SBM: 2000. CD-ROM

BRASIL. Ministério da Agricultura - Departamento de Pesquisa Agropecuária. Levantamento de reconhecimento dos solos do Estado do Rio Grande do Sul. Recife: 1973. 431 p. (Boletim Técnico, 30).

CALDEIRA, M. V. W. Quantificação da biomassa e do conteúdo de nutrientes em diferentes procedências de acácia-negra (Acacia mearnsii De Wild.). 1998. $96 \mathrm{f}$. Dissertação (Mestrado em Engenharia Florestal) Universidade Federal de Santa Maria, Santa Maria, 1998.
CALDEIRA, M. V. W. et al. Ciclagem de nutrientes em Acacia mearnsii De Wild. V quantificação do conteúdo de nutrientes na biomassa aérea de Acacia mearnsii De Wild. procedência australiana. Ciência Rural, v. 30, n. 6, p. 977982, 2000.

CALDEIRA, M. V. W. et al. Concentração e redistribuição de nutrientes nas folhas e no folhedo em povoamento comercial de Acacia mearnsii De Wild. plantado no Rio Grande do Sul. Ciência Florestal, v. 9, n. 1, p. 19-24, 1999b.

CALDEIRA, M. V. W. et al. Estimativa do conteúdo de nutrientes em um povoamento jovem de Acacia mearnsii De Wild. estabelecido na região sul do Brasil. Floresta, v. 29, n. 1/2, p. 53-65, 1999a.

CALDEIRA, M. V. W.; PEREIRA, J. C.; SCHUMACHER, M. V. Comparação entre as concentração de nutrientes nas folhas e no folhedo em procedências de Acacia mearnsii De Wild. Revista Árvore, v. 23, n. 4, p. 489-492, 1999 c.

CALDEIRA, M. V. W.; SCHUMACHER, M. V.; SANTOS, E. M. Conteúdo de nutrientes em uma procedência de Acacia mearnsii plantada no Rio Grande do Sul. Boletim de Pesquisa Florestal, n. 42, p. 105-121, 2001.

DALLAGO, J. S. Utilização da cinza de biomassa de caldeira como fonte de nutrientes no crescimento de plantas de acácia-negra (Acacia mearnsii De Wild.). 2000. 64 f. Dissertação (Mestrado em Engenharia Florestal) - Universidade Federal de Santa Maria, Santa Maria, 2000.

EMPRESA BRASILEIRA DE PESQUISA AGROPECUÁRIA - EMBRAPA. Sistema brasileiro de classificação levantamento de solos. Rio de Janeiro: 1999. $412 \mathrm{p}$.

HIGA, A. R. et al. Desarrollo de sistemas de produccoón para acacia negra (Acacia mearnsii De Wild.). In: CONGRESSO LATINOAMERICANO IUFRO - O manejo sustentable de los recursos forestales, desafío del siglo XXI, 1., 1998, Valdivia. Resumos... Valdivia: IUFRO, 1998. CD-ROM

INSTITUTO DE PESQUISAS AGRONÔMICAS IPAGRO. Atlas agroclimático do Estado do Rio Grande do Sul. Porto Alegre: 1989. 3 v.

KOEHLER, C. W. Variação estacional da deposição de serapilheira e de nutientes em povoamentos de Pinus taeda na região de Ponta Grossa-PR. 1989. $148 \mathrm{f}$. Tese (Doutorado em Ciências Florestais) - Universidade Federal do Paraná, Curitiba, 1989.

R. Árvore, Viçosa-MG, v.27, n.1, p.9-14, 2003 
KRAMER, P. J.; KOZLOWSKI, T. T. Physiology of woody plants. New York: Academic Press, 1979. 811 p.

LIMA, W. P. Impacto ambiental do eucalipto. 2.ed. São Paulo: USP, 1996. p. 139-168.

MARSCHNER, H. Mineral nutrition of higher plants. San Diego: Academic Press, 1997. 889 p.

MORENO, J. A. Clima do Rio Grande do Sul. Porto Alegre: Secretaria da Agricultura, 1961. 42 p.

PEREIRA, J. C. et al. Exportação de nutrientes em um povoamento de Acacia mearnsii De Wild. em idade de corte. In: CICLO DE ATUALIZAÇÃO FLORESTAL DO CONESUL, 1., Santa Maria-RS. Anais... Santa Maria: UFSM, 1999. p. $158-164$.

PEREIRA, J. C. et al. Estimativa do conteúdo de nutrientes em um povoamento de de Acacia mearnsii De Wild. no Rio Grande do Sul - Brasil. Revista Árvore, v. 24, n. 2, p. 193 199, 2000.

POGGIANI, F. et al. Exportação de biomassa e nutrientes através da explotação dos troncos e das copas de um povoamento de Eucalyptus saligna. IPEF, v. 25, p. 37-39, 1983.
TEDESCO, M. J. et al. Análise de solos, plantas e outros materiais. Porto Alegre: Universidade Federal do Rio Grande do Sul, 1995. 174 p. (Boletim Técnico, 5).

VALERI, S. V.; REISSMANN, C. B.; SANTOS-FILHO, A. Exportação de nutrientes de povoamentos de Pinus taeda L. desbastados em diferentes idades. Floresta, v. 19, n. 1/2, p. 62-68, 1989.

VAN DEN DRIESSCHE, R. Prediction of mineral status of trees by foliar analysis. The Botanical Review, v. 40, p. 347-394, 1984.

VEZZANI, F. M. Aspectos nutricionais de povoamentos puros e misto de Eucalyptus saligna Smith e de Acacia mearnsii De Wild. 1997. 97 f. Dissertação (Mestrado em Ciências do Solo) - Universidade Federal do Rio Grande do Sul, Porto Alegre, 1997.

YONG, H. E.; CARPENTER, P. N. Sampling variation of nutrient element content within and betwen on trees of the same species. In: OSLO BIOMASS STUDIES, 1976, Oslo. Proceedings... Oslo: 1976. p. 75-90. 